\title{
Entanglement manipulation via dynamics in multiple quantum spin systems
}

\author{
Andrea Casaccino \\ Computer Architecture Group Lab, Information Engineering Department, University of Siena, 53100 Siena, Italy \\ Research Laboratory of Electronics, Massachusetts Institute of Technology, Cambridge, 020139, USA \\ Stefano Mancini \\ School of Science and Technology, University of Camerino, 62032 Camerino, Italy \\ and INFN, Sezione di Perugia, 06123 Perugia, Italy \\ Simone Severini \\ Department of Physics and Astronomy, University College London, WC1E 6BT London, United Kingdom
}

\begin{abstract}
We study manipulation of entanglement between two identical networks of quantum mechanical particles. Firstly, we reduce the problem of entanglement transfer to the problem of quantum state transfer. Then, we consider entanglement concentration and purification based on free dynamics on the networks and local measurements on the vertices. By introducing an appropriate measure of efficiency, we characterize the performance of the protocol. We give evidence that such a measure does not depend on the network topology, and we estimate the contribution given by the number of entangled pairs initially shared by the two networks.
\end{abstract}

PACS numbers: 03.67.Mn, 02.10.Ox

\section{INTRODUCTION}

Networks of quantum mechanical particles play a fundamental role in extending quantum information processing to multipartite settings and in foreseeing the realization of distributed protocols for multi-users devices [1].

In the present work we study manipulation of entanglement between quantum networks and attempt to isolate useful graph-theoretic properties in relation to the dynamics of entanglement. Specifically, we analyze the problem of entanglement transfer through networks and then we relate its setting to the fidelity problem associated with state transfer.

Our goal is to study entanglement concentration and purification, when the evolution of the system involves two networks of spin-half particles with $X Y$-type interaction. Entanglement concentration (and, more generally, purification) is among the most relevant tasks in quantum information processing [2]. It is a process extracting strongly entangled pairs out of initially weakly entangled ones, only by mean of local operations and classical communication. Most of the known protocols involve control-not operations. In a similar way, multipartite measurements and classical communication are used to propagate entanglement in certain type of quantum networks [3, 4]. Entanglement concentration has also been obtained by exploiting the effects of quantum statistics of indistinguishable particles [5]. A streaming (sequential) protocol for universal entanglement concentration has recently appeared in [6], where a number of input systems are processed in sequence, and perfect entangled pairs are obtained at the end of the protocol.

Following [7], we simply exploit free dynamics on the networks and local measurements on the sites. Somehow contrarily to a claim proposed in [7], we show that this method works reasonably well also in the case of $X Y$ type interaction. We consider generic network topologies other than linear spin chains and we introduce an efficiency measure to characterize the performance of the protocol. It will turn out that, at least in the cases considered, the structure of the networks only affects the time at which the maximum efficiency is attained.

The topic of this paper fits into a growing literature. State and energy transfer in spin systems are now areas of extensive study [8] (see also [9]). Protocols for creating entanglement between distant sites in a quantum network have been designed in [10], where the network considered is a lattices, whose vertices share pure, nonmaximally entangled pairs of qubits. The problem of preparing maximally entangled states between different sites in a distributed quantum system is related to classical percolation in statistical mechanics 11]. In [12], the study of entanglement percolation is extended to structures with precise statistical properties but unknown local topology. In [13], entanglement percolation is used for long-distance singlet generation in networks with adjacent vertices connected by partially entangled bipartite mixed states.

The structure of the paper is as follows. In Section II, we introduce the considered networks model relying on the basic $X Y$-type interaction. In Section III, we give rigorous results for the problem of entanglement transfer in quantum networks by considering the single excitation model. The quality of the entanglement transmission is then related to the fidelity achieved between the considered nodes, therefore extending the cases studied in 9]. In Section IV, we discuss how to enhance the entanglement of a pure states through free dynamics and local measurements on the nodes. We introduce an efficiency measure for our protocols, by considering all possible measurement outcomes and the corresponding prob- 
abilities. Finally, in Section V, we show how the protocol works in the case of mixed entangled states by considering Werner mixtures [14]. Likewise Section IV, the efficiency of the procedure will be related to the minimal number of initially entangled pairs necessary to successfully complete the protocol. Conclusions are drawn in Section VI, where we summarize the results and mention potential physical realizations.

\section{NETWORKS MODEL}

Let $G=(V, E)$ be a simple undirected graph (that is, without loops or parallel edges), with set of vertices $V(G)$ and set of edges $E(G)$. The adjacency matrix of $G$ is denoted by $A(G)$ and defined by $[A(G)]_{i j}=1$, if $i j \in E(G)$; $[A(G)]_{i j}=0$ if $i j \notin E(G)$. The adjacency matrix is a useful tool to describe a network of $n$ spin-half quantum particles. The particles are usually associated to the vertices of $G$, while the edges of $G$ represent their allowed couplings. If one considers the $X Y$ interaction model, then $\{i, j\} \in E(G)$ means that the particles $i$ and $j$ interact by the Hamiltonian $[H(G)]_{i j}=-\left(X_{i} X_{j}+Y_{i} Y_{j}\right)$, where $X_{i}, Y_{i}$ are the Pauli operators of the $i$-th particle (here we consider unit negative coupling constant). Thus, the Hamiltonian of the whole network reads

$$
H(G)=-\frac{1}{2} \sum_{i \neq j}[A(G)]_{i j}\left(X_{i} X_{j}+Y_{i} Y_{j}\right)
$$

and it acts on the Hilbert space $\mathcal{H}=\left(\mathbb{C}^{2}\right)^{\otimes n}$.

In the following we will consider two networks with underlying graph $G_{A}$ and $G_{B}$ having, for the sake of simplicity, an equal number of nodes $n$. Then, if we apply $X Y$ model we have two Hamiltonians

$$
\begin{aligned}
& H\left(G_{A}\right)=-\frac{1}{2} \sum_{i, j}^{n}\left[A\left(G_{A}\right)\right]_{i j}\left(X_{A_{i}} X_{A_{j}}+Y_{A_{i}} Y_{A_{j}}\right), \\
& H\left(G_{B}\right)=-\frac{1}{2} \sum_{i, j}^{n}\left[A\left(G_{B}\right)\right]_{i j}\left(X_{B_{i}} X_{B_{j}}+Y_{B_{i}} Y_{B_{j}}\right) .
\end{aligned}
$$

\section{ENTANGLEMENT TRANSFER}

Let $A$ and $B$ be two networks sharing a pair of qubits in a maximally entangled state

$$
\frac{1}{\sqrt{2}}(|0\rangle|1\rangle+|1\rangle|0\rangle) .
$$

Suppose that the entangled qubits are in the $i$-th site of the network $A$ and in the $k$-th site of the network $B$. We assume that all the remaining qubits are in the ground state. Then the state for the joint system $A+B$ will be

$$
\frac{1}{\sqrt{2}}\left(|0\rangle_{A}|k\rangle_{B}+|i\rangle_{A}|0\rangle_{B}\right)
$$

where $|i\rangle_{A}$ denotes the state $|0\rangle_{1}|0\rangle_{2} \ldots|1\rangle_{i} \ldots|0\rangle_{n-1}|0\rangle_{n}$ for the network $A$; a similar notation is used for the network $B$.

The evolution of the whole system will be governed by the Hamiltonians (2). Hence, the global evolution of the two networks will lead to the state

$$
\begin{aligned}
& \frac{1}{\sqrt{2}}\left(|0\rangle_{A} e^{\iota H\left(G_{B}\right) t}|k\rangle_{B}+e^{\iota H\left(G_{A}\right) t}|i\rangle_{A}|0\rangle_{B}\right) \\
& =\frac{1}{\sqrt{2}}\left(|0\rangle_{A}\left|\varphi_{k}\right\rangle_{B}+\left|\psi_{i}\right\rangle_{A}|0\rangle_{B}\right),
\end{aligned}
$$

where $\left|\psi_{i}\right\rangle_{A}=e^{\iota H\left(G_{A}\right) t}|i\rangle_{A}$ and $\left|\varphi_{k}\right\rangle_{B}=e^{\iota H\left(G_{B}\right) t}|k\rangle_{B}$.

To see how entanglement propagates from the sites $i$ and $k$, we evaluate the concurrence [15] between two generic sites $j$ and $l$ (different from $i, k$ ). To this end, we have to consider the following density matrix $\rho_{A B}$ :

$$
\begin{aligned}
\rho_{A B} & =\frac{1}{2} \operatorname{Tr}_{j}\left\{|0\rangle_{A}\langle 0|\right\} \otimes \operatorname{Tr}_{\ell}\left\{\left|\varphi_{k}\right\rangle_{B}\left\langle\varphi_{k}\right|\right\} \\
& +\frac{1}{2} \operatorname{Tr}_{j}\left\{|0\rangle_{A}\left\langle\psi_{i}\right\} \mid \otimes \operatorname{Tr}_{\ell}\left\{\left|\varphi_{k}\right\rangle_{B}\langle 0|\right\}\right. \\
& +\frac{1}{2} \operatorname{Tr}_{j}\left\{\left|\psi_{i}\right\rangle_{A}\langle 0|\right\} \otimes \operatorname{Tr}_{\ell}\left\{|0\rangle_{B}\left\langle\varphi_{k}\right|\right\} \\
& +\frac{1}{2} \operatorname{Tr}_{j}\left|\psi_{i}\right\rangle_{A}\left\langle\psi_{i}\right| \otimes \operatorname{Tr}_{\ell\{}\left\{|0\rangle_{B}\langle 0|\right\},
\end{aligned}
$$

where $\operatorname{Tr}_{j}$ denotes the trace overall qubits but the $j$-th one.

For a single pure excitation of a spin-half network, the wave functions can be expanded as

$$
|\psi\rangle=\alpha_{1}|1\rangle+\ldots+\alpha_{n}|n\rangle
$$

or

$$
|\varphi\rangle=\beta_{1}|1\rangle+\ldots+\beta_{n}|n\rangle .
$$

In this way it is possible to express the density matrix $\rho_{A B}$ as a function of the complex coefficients $\alpha$ and $\beta$ :

$$
\rho_{A B}=\frac{1}{2}\left(\begin{array}{cccc}
\sum_{i \neq j}^{n}\left|\alpha_{i}\right|^{2}+\sum_{k \neq \ell}^{n}\left|\beta_{k}\right|^{2} & 0 & 0 & 0 \\
0 & \left|\beta_{\ell}\right|^{2} & \alpha_{j}^{*} \beta_{\ell} & 0 \\
0 & \alpha_{j} \beta_{\ell}^{*} & \left|\alpha_{j}\right|^{2} & 0 \\
0 & 0 & 0 & 0
\end{array}\right) .
$$

For the single excitation model including both networks with Hamiltonians (2), the $\rho_{A B} \check{\rho}_{A B}$ matrix is

$$
\begin{aligned}
& \rho_{A B} \check{\rho}_{A B} \\
& =\frac{1}{4}\left(\begin{array}{cccc}
0 & 0 & 0 & 0 \\
0 & 2\left|\alpha_{j}\right|^{2}\left|\beta_{\ell}\right|^{2} & \left|\alpha_{j}\right|^{2}\left(\alpha_{j}^{*} \beta_{\ell}+\alpha_{j} \beta_{\ell}^{*}\right) & 0 \\
0 & \left|\beta_{\ell}\right|^{2}\left(\alpha_{j}^{*} \beta_{\ell}+\alpha_{j} \beta_{\ell}^{*}\right) & 2\left|\alpha_{j}\right|^{2}\left|\beta_{\ell}\right|^{2} & 0 \\
0 & 0 & 0 & 0
\end{array}\right) .
\end{aligned}
$$

Here, accordingly to [15]

$$
\check{\rho}_{A B}=\left(Y_{A} \otimes Y_{B}\right) \rho_{A B}^{*}\left(Y_{A} \otimes Y_{B}\right),
$$


where $\rho_{A B}^{*}$ is the complex conjugate of $\rho_{A B}$ [15].

The concurrence between two qubits $j$ and $l$ is defined as [15]

$$
C_{j, l}(\rho)=\max \left(0, \sqrt{\lambda_{1}}-\sqrt{\lambda_{2}}-\sqrt{\lambda_{3}}-\sqrt{\lambda_{4}}\right),
$$

where $\lambda_{1}, \lambda_{2}, \lambda_{3}$, and $\lambda_{4}$ are the eigenvalues of $\rho_{A B} \check{\rho}_{A B}$ in the decreasing order.

Explicitly,

$$
C_{j, l}=\left|\alpha_{j}\right|\left|\beta_{\ell}\right|
$$

It turns out that the concurrence is equal to the product of the fidelity reached during the transmission of a single excitation in each network. As it is expected, if there is perfect state transfer between particle $i$ and particle $j$ on $A$ as well as between particles $k$ and $l$ on $B$, entanglement is perfectly transferred from the pair $i, k$ to the pair $j, l$.

\section{ENTANGLEMENT CONCENTRATION}

We now consider two identical networks sharing pairs of qubits in a pure, non- maximally entangled state and look for a possibility of enhancing the amount of such entanglement.

\section{A. Two qubits networks}

In the simplest configuration we may consider two sites on each network connected by an edge. These networks are simply two graphs consisting of a single edge. Then, the resulting Hamiltonians $H_{A}$ and $H_{B}$ correspond to the adjacency matrices

$$
A\left(G_{A, B} ; P_{2}\right)=\left(\begin{array}{cc}
0 & 1 \\
1 & 0
\end{array}\right),
$$

where $P_{n}$ denotes an open chain of length $n-1$. Let the initial state of qubits $A_{1}$ and $B_{1}$ be

$$
|\psi\rangle=\cos (\theta)|0\rangle_{A_{1}}|0\rangle_{B_{1}}+\sin (\theta)|1\rangle_{A_{1}}|1\rangle_{B_{1}}
$$

and the initial state of qubits $A_{2}$ and $B_{2}$ be $|0\rangle_{A_{2}}|0\rangle_{B_{2}}$. The two networks will evolve independently, so that the whole state

$$
\begin{aligned}
|\Psi\rangle & =\cos (\theta)|0\rangle_{A_{1}}|0\rangle_{B_{1}}|0\rangle_{A_{2}}|0\rangle_{B_{2}} \\
& +\sin (\theta)|1\rangle_{A_{1}}|1\rangle_{B_{1}}|0\rangle_{A_{2}}|0\rangle_{B_{2}}
\end{aligned}
$$

will have the following general form at time $t$ :

$$
\begin{aligned}
|\Psi(t)\rangle & =\cos (\theta)|0\rangle_{A_{1}}|0\rangle_{A_{2}}|0\rangle_{B_{1}}|0\rangle_{B_{2}} \\
& +\sin (\theta) \cos ^{2}(t)|1\rangle_{A_{1}}|0\rangle_{A_{2}}|1\rangle_{B_{1}}|0\rangle_{B_{2}} \\
& +\sin (\theta) \cos (t) \sin (t)|1\rangle_{A_{1}}|0\rangle_{A_{2}}|0\rangle_{B_{1}}|1\rangle_{B_{2}} \\
& +\sin (\theta) \cos (t) \sin (t)|0\rangle_{A_{1}}|1\rangle_{A_{2}}|1\rangle_{B_{1}}|0\rangle_{B_{2}} \\
& +\sin (\theta) \sin ^{2}(t)|0\rangle_{A_{1}}|1\rangle_{A_{2}}|0\rangle_{B_{1}}|1\rangle_{B_{2}} .
\end{aligned}
$$

Actually, given the evolution, also the qubits $A_{2}$ and $B_{2}$ become entangled with $A_{1}$ and $B_{1}$. Local measurements on $A_{2}$ and $B_{2}$ may concentrate the entanglement available at time $t$ on qubits $A_{1}$ and $B_{1}$, possibly increasing the initial amount. Suppose that we locally measure $Z_{A_{2}} \otimes Z_{B_{2}}$. For outcomes 00, the state in Eq. (16) will be projected onto $|0\rangle_{A_{2}}|0\rangle_{B_{2}}$. Hence, the state $\sigma=|\Psi(t)\rangle\langle\Psi(t)|$ changes into

$$
\sigma \mapsto \rho_{A_{1} B_{1}} \otimes|0\rangle_{A_{2}}\langle 0|\otimes| 0\rangle_{B_{2}}\langle 0|,
$$

where

$$
\rho_{A_{1} B_{1}}=\frac{\tilde{\rho}_{A_{1} B_{1}}}{\operatorname{Tr}_{A_{1} B_{1}}\left\{\tilde{\rho}_{A_{1} B_{1}}\right\}},
$$

and

$$
\tilde{\rho}_{A_{1} B_{1}}={ }_{A_{2}}\left\langle\left. 0\right|_{B_{2}}\langle 0|\sigma| 0\rangle_{B_{2}} \mid 0\right\rangle_{A_{2}} .
$$

It results

$$
\begin{aligned}
\tilde{\rho}_{A_{1} B_{1}} & ={ }_{A_{2}}\left\langle\left. 0\right|_{B_{2}}\langle 0|| \Psi(t)\rangle\langle\Psi(t)|| 0\rangle_{B_{2}} \mid 0\right\rangle_{A_{2}} \\
& =\left(\cos (\theta)|0\rangle_{A_{1}}|0\rangle_{B_{1}}+\sin (\theta) \cos ^{2}(t)|1\rangle_{A_{1}}|1\rangle_{B_{1}}\right) \\
& \left(\operatorname { c o s } ( \theta ) _ { A _ { 1 } } \left\langle\left.0\right|_{B_{1}}\langle 0|+\sin (\theta) \cos ^{2}(t)_{A_{1}}\left\langle\left. 1\right|_{B_{1}}\langle 1|\right) .\right.\right.
\end{aligned}
$$

The probability of this transformation is

$$
P_{00}(t)=\operatorname{Tr}_{A_{1} B_{1}}\left\{\tilde{\rho}_{A_{1} B_{1}}\right\}=\cos ^{2}(\theta)+\sin ^{2}(\theta) \cos ^{4}(t) .
$$

Notice that the state (18) with (19) and (21) is quite generally entangled with a concurrence possibly higher then the initial state (16). On the contrary, it is easy to check that the state resulting from the projection of Eq.(16) onto $|0\rangle_{A_{2}}|1\rangle_{B_{2}}$, or $|1\rangle_{A_{2}}|0\rangle_{B_{2}}$, or $|1\rangle_{A_{2}}|1\rangle_{B_{2}}$ (corresponding to outcomes $01,10,11$ respectively) is separable, hence with zero concurrence.

Since the enhancement of the initial entanglement is determined by the measurement outcomes, we have to account for their respective probabilities in order to evaluate the performance of the protocol. Then, we define the efficiency of the protocol as the average of the increments of entanglement weighted by the respective probabilities, that is,

$$
E:=\sum_{o} P_{o} \Delta_{o} C
$$

Here the index $o$ runs over all possible measurement outcomes, each occurring with probability $P_{o}$. Moreover

$$
\Delta_{o} C:= \begin{cases}\left(C_{o}-C\right), & \text { if } C_{o}>C ; \\ 0, & \text { if } C_{o} \leq C .\end{cases}
$$

Here $C$ is the concurrence of $\rho_{A_{1} B_{1}}$ and $C_{o}$ the concurrence of $\rho_{A_{1} B_{1}}^{\prime}$ conditioned to the outcome $o$.

In our case the concurrence for the outcome 00 reads

$$
C_{00}=\frac{2 \sin (\theta) \cos (\theta) \cos ^{2}(t)}{\cos ^{2}(\theta)+\sin ^{2}(\theta) \cos ^{4}(t)}
$$


thus the efficiency simply results

$$
\begin{aligned}
& E(\theta, t)=P_{00} \Delta_{00} C \\
& =2 \sin (\theta) \cos (\theta)\left(\cos ^{2}(t)-\cos ^{2}(\theta)-\sin ^{2}(\theta) \cos ^{4}(t)\right) .
\end{aligned}
$$

Now taking $\frac{\partial E(\theta, t)}{\partial t}=0$ we get the optimal time (maximizing (25) ) as

$$
\cos ^{2}(t)=\frac{1}{2 \sin ^{2}(\theta)}
$$

By substituting it back to Eq.(25) we arrive at the maximum efficiency written as solely function of the variable $\theta$

$$
E(\theta)=\frac{\cos (\theta)}{2 \sin (\theta)}\left(1-4 \cos ^{2}(\theta) \sin ^{2}(\theta)\right) .
$$

The behavior of this quantity is depicted in Fig. (11).

Let us now consider the case of two initially entangled pairs. Let Eq. (14) be the initial state of qubits $A_{1}$ and $B_{1}$ as well as qubits $A_{2}$ and $B_{2}$. Write $v_{A_{i} B_{i}}=|\psi\rangle\langle\psi|$, then the initial state of the networks simply results

$$
v_{A_{1} B_{1}} \otimes v_{A_{2} B_{2}}
$$

and it evolves into

$$
\sigma=e^{-\iota H_{A} t} e^{-\iota H_{B} t}\left(v_{A_{1} B_{1}} \otimes v_{A_{2} B_{2}}\right) e^{\iota H_{B} t} e^{\iota H_{A} t} .
$$

Now let us consider local measurements of the Pauli observables $Z_{A_{2}} \otimes Z_{B_{2}}$, i.e., only one out of the two initial pairs is locally measured.

For outcome $a_{2} b_{2}$ (with $a_{2}, b_{2} \in\{0,1\}$ ) the above state will be projected onto $\left|a_{2}\right\rangle_{A_{2}}\left|b_{2}\right\rangle_{B_{2}}$. Hence,

$$
\sigma \mapsto \rho_{A_{1} B_{1}} \otimes\left|a_{2}\right\rangle_{A_{2}}\left\langle a_{2}|\otimes| b_{2}\right\rangle_{B_{2}}\left\langle b_{2}\right|,
$$

where

$$
\rho_{A_{1} B_{1}}=\frac{\tilde{\rho}_{A_{1} B_{1}}}{\operatorname{Tr}_{A_{1} B_{1}}\left\{\tilde{\rho}_{A_{1} B_{1}}\right\}},
$$

and

$$
\tilde{\rho}_{A_{1} B_{1}}=\left\langle a_{2} b_{2}|\sigma| b_{2} a_{2}\right\rangle .
$$

Outcome $a_{2} b_{2}=00$ occurs at time $t$ with probability

$$
\begin{aligned}
P_{00}(t) & =\operatorname{Tr}_{A_{1} B_{1}}\left\{\tilde{\rho}_{A_{1} B_{1}}\right\} \\
& =\frac{1}{2} \cos ^{2}(\theta)\left(2 \cos ^{2}(\theta)-\sin ^{2}(\theta)-\sin ^{2}(\theta) \cos (4 t)\right)
\end{aligned}
$$

and the corresponding conditional state results (in matrix form)

$$
\begin{aligned}
& \tilde{\rho}_{A_{1} B_{1}}(t)= \\
& \left(\begin{array}{cccc}
\cos ^{4}(\theta) & 0 & 0 & \cos ^{3}(\theta) \sin (\theta) \cos (2 t) \\
0 & 0 & 0 & 0 \\
0 & 0 & 0 & 0 \\
\cos ^{3}(\theta) \sin (\theta) \cos (2 t) & 0 & 0 & \cos ^{2}(\theta) \sin ^{2}(\theta) \cos ^{2}(2 t)
\end{array}\right) .
\end{aligned}
$$

Its concurrence reads

$$
C_{00}(t)=\frac{4 \sin (\theta) \cos (\theta) \cos (2 t)}{2 \cos ^{2}(\theta)-\sin ^{2}(\theta)-\sin ^{2}(\theta) \cos (4 t)} .
$$

Outcomes $a_{2} b_{2}=11$ occurs at time $t$ with probability

$$
\begin{aligned}
P_{11}(t) & =\operatorname{Tr}_{A_{1} B_{1}}\left\{\tilde{\rho}_{A_{1} B_{1}}\right\} \\
& =\frac{1}{2} \sin ^{2}(\theta)\left(\cos ^{2}(\theta)+-2 \sin ^{2}(\theta)+\cos ^{2}(\theta) \cos (4 t)\right)
\end{aligned}
$$

and the corresponding conditional state results (in matrix form)

$$
\begin{aligned}
& \tilde{\rho}_{A_{1} B_{1}}(t)= \\
& \left(\begin{array}{cccc}
\cos ^{2}(\theta) \sin ^{2}(\theta) \cos ^{2}(2 t) & 0 & 0 & \cos (\theta)^{3} \sin (\theta) \cos (2 t) \\
0 & 0 & 0 & 0 \\
0 & 0 & 0 & 0 \\
\cos (\theta) \sin ^{3}(\theta) \cos (2 t) & 0 & 0 & \sin ^{4}(\theta)
\end{array}\right)
\end{aligned}
$$

Its concurrence reads

$$
C_{11}(t)=\frac{4 \sin (\theta) \cos (\theta) \cos (2 t)}{\cos ^{2}(\theta)-2 \sin ^{2}(\theta)+\cos ^{2}(\theta) \cos (4 t)} .
$$

Since the other outcomes $\left(a_{2} b_{2}=01,10\right)$ give separable conditional states, the efficiency in Eq.(22) becomes

$$
\begin{aligned}
E(\theta, t) & =\cos ^{4}(\theta) \sin ^{4}(\theta) \\
& \times\left(\cos ^{2}(\theta)-2 \sin ^{2}(\theta)-2 \cos (2 t)+\cos ^{2}(\theta) \cos (4 t)\right) \\
& \times\left(2 \cos ^{2}(\theta)-\sin ^{2}(\theta)-2 \cos (2 t)-\sin ^{2}(\theta) \cos (4 t)\right) \\
& -2 \cos ^{5}(\theta) \sin (\theta) \cos (4 t)
\end{aligned}
$$

Now, by taking $\frac{\partial E(\theta, t)}{\partial t}=0$ we get the optimal time (maximizing (39) ) as $t=\pi$. By substituting it back to Eq. (39) we arrive at the maximum efficiency written as solely function of the variable $\theta$

$$
E(\theta)=2 \cos ^{4}(\theta) \sin (\theta)\left(8 \sin ^{7}(\theta)-\cos (\theta)\right)
$$

The behavior of this quantity is depicted in Fig. (1) for $\pi / 4 \leq \theta \leq \pi / 2$.

\section{B. Networks with more than two qubits}

In the previous subsection we have seen that having initially a single entangled pure pair suffices to achieve entanglement concentration. However, looking at Fig. (11), we can conclude that having initially two entangled pure pairs give to the protocol a better performance. From now on we are going to consider a number of initially entangled pure pairs equal to the number of vertices in the network. For $n$ vertices, consider the initial state of qubits $A_{i}$ and $B_{i}$ to be (14). Writing $v_{A_{i} B_{i}}=\left|\psi_{i}\right\rangle\left\langle\psi_{i}\right|$, the initial state of the system is then

$$
V_{12 \ldots n}=v_{A_{1} B_{1}} \otimes v_{A_{2} B_{2}} \otimes \ldots \otimes v_{A_{n} B_{n}}
$$


The time-evolution gives

$$
\sigma=e^{-\iota H_{A} t} e^{-\iota H_{B} t} V_{12 \ldots n} e^{\iota H_{B} t} e^{\iota H_{A} t} .
$$

Now we measure with respect to the Pauli observables $\left(Z_{A_{2}} \otimes \ldots \otimes Z_{A_{n}}\right) \otimes\left(Z_{B_{2}} \otimes \ldots \otimes Z_{B_{n}}\right)$, i.e., $n-1$ out of initially $n$ pairs are locally subjected to measurement.

When we obtain $a_{2} \ldots a_{n} b_{2} \ldots b_{n}$, where each variable is either zero or one, the above state will be projected onto $\left|a_{2}\right\rangle_{A_{2}} \ldots\left|a_{n}\right\rangle_{A_{n}}\left|b_{2}\right\rangle_{B_{2}} \ldots\left|b_{n}\right\rangle_{B_{n}}$. Then

$$
\begin{aligned}
\sigma \mapsto \rho_{A_{1} B_{1}} & \otimes\left|a_{2}\right\rangle_{A_{2}}\left\langle a_{2}|\otimes \ldots \otimes| a_{n}\right\rangle_{A_{n}}\left\langle a_{n}\right| \\
& \otimes\left|b_{2}\right\rangle_{B_{2}}\left\langle b_{2}|\otimes \ldots \otimes| b_{n}\right\rangle_{B_{n}}\left\langle b_{n}\right|,
\end{aligned}
$$

where

$$
\rho_{A_{1} B_{1}}=\frac{\tilde{\rho}_{A_{1} B_{1}}}{\operatorname{Tr}_{A_{1} B_{1}}\left\{\tilde{\rho}_{A_{1} B_{1}}\right\}},
$$

and

$$
\tilde{\rho}_{A_{1} B_{1}}=\left\langle a_{2} \ldots a_{n} b_{2} \ldots b_{n}|\sigma| b_{n} \ldots b_{2} a_{n} \ldots a_{2}\right\rangle .
$$

By taking into account all $4^{n-1}$ possible outcomes we can then calculate the efficiency given in Eq. (22).

Let us now consider $n=3$ and the two networks in an open chain configuration. Then, the Hamiltonians $H_{A}$ and $H_{B}$ come from the following adjacency matrices

$$
A\left(G_{A, B} ; P_{3}\right)=\left(\begin{array}{ccc}
0 & 1 & 0 \\
1 & 0 & 1 \\
0 & 1 & 0
\end{array}\right)
$$

The efficiency is maximized by $t=k \pi / \sqrt{2}, k \in \mathbb{N}$ giving

$$
E(\theta)=5 \cos ^{2}(\theta) \sin ^{2}(\theta)\left(1-2 \cos ^{2}(\theta)\right),
$$

where $\pi / 4 \leq \theta \leq \pi / 2$.

The same efficiency can be obtained by considering the dynamics resulting from the fully connected three qubits networks. The related Hamiltonians $H_{A}$ and $H_{B}$ are

$$
A\left(G_{A, B} ; K_{3}\right)=\left(\begin{array}{ccc}
0 & 1 & 1 \\
1 & 0 & 1 \\
1 & 1 & 0
\end{array}\right)
$$

where $K_{n}$ is the fully connected graph with $n$ vertices. However, in this case, the expression (47) is obtained at time $t=k \pi, k \in \mathbb{N}$. The quantity (47) is represented in Fig. (11).

Practically, the change of the underlying graph associated to the network only affects the time of the dynamics, but it does not affect the expression of the maximum efficiency. We conjecture (supported by numerical evidence) that this is also true for networks having more than three nodes, provided that there are no isolated nodes. In fact, the protocol involves entangled pairs shared by single nodes of each network. Since we consider a number of initially entangled pairs equal to the number of nodes in a network, two isolated nodes that share an entangled pair would constitute a separate system. Then, the maximum efficiency only depends on the number of nodes and so on the total number of initially entangled pairs.

Let us now consider $n=4$ and suppose that the two networks $A$ and $B$ are both in a closed chain configuration (a cycle). Then, the Hamiltonians $H_{A}$ and $H_{B}$ result from the following adjacency matrices

$$
A\left(G_{A, B} ; C_{4}\right)=\left(\begin{array}{cccc}
0 & 1 & 0 & 1 \\
1 & 0 & 1 & 0 \\
0 & 1 & 0 & 1 \\
1 & 0 & 1 & 0
\end{array}\right),
$$

where $C_{n}$ is the closed chain of length $n-1$. In this case the maximum efficiency is obtained for $t=k \pi, k \in \mathbb{N}$ and it reads

$E(\theta)=\frac{1}{16} \cos (\theta) \sin ^{3}(\theta)(8 \cos (4 \theta)-3 \cos (6 \theta)-29 \cos (2 \theta)+8)$,

where $\pi / 4 \leq \theta \leq \pi / 2$. Such efficiency is attained at $t=\pi / 2+k \pi, k \in \mathbb{N}$, in the case of fully connected configurations.

The efficiency (50) is represented in Fig. (11).

Let us finally consider the case of $n=5$. We chose Hamiltonians $H_{A}$ and $H_{B}$ coming from the following adjacency matrices

$$
A\left(G_{A, B} ; C_{5}\right)=\left(\begin{array}{ccccc}
0 & 1 & 0 & 0 & 1 \\
1 & 0 & 1 & 0 & 0 \\
0 & 1 & 0 & 1 & 0 \\
0 & 0 & 1 & 0 & 1 \\
1 & 0 & 0 & 1 & 0
\end{array}\right)
$$

The maximum efficiency results

$E(\theta)=\frac{1}{4} \cos (\theta) \sin ^{3}(\theta)(\cos (4 \theta)-\cos (6 \theta)-13 \cos (2 \theta)+1)$,

where $\pi / 4 \leq \theta \leq \pi / 2$. This is obtained for $t=k \pi, k \in \mathbb{N}$. The efficiency (52) is represented in Fig 1.

\section{ENTANGLEMENT PURIFICATION}

We shall consider pairs of qubits in networks $A$ and $B$ initially in a mixed entangled state. We then determine conditions for enhancing such entanglement. Specifically, we consider the initial state of qubits $A_{i} B_{i}$ to be the Werner mixture [14] written as

$$
\begin{aligned}
w_{A_{i} B_{i}} & =f\left|\Phi^{+}\right\rangle_{A_{i} B_{i}}\left\langle\Phi^{+}\right|+\frac{1-f}{3}\left(\left|\Phi^{-}\right\rangle_{A_{i} B_{i}}\left\langle\Phi^{-}\right|\right. \\
& \left.+\left|\Psi^{+}\right\rangle_{A_{i} B_{i}}\left\langle\Psi^{+}|+| \Psi^{-}\right\rangle_{A_{i} B_{i}}\left\langle\Psi^{-}\right|\right),
\end{aligned}
$$

where

$$
\begin{aligned}
\left|\Phi^{ \pm}\right\rangle_{A_{i} B_{i}} & =\frac{|0\rangle_{A_{i}}|0\rangle_{B_{i}} \pm|1\rangle_{A_{i}}|1\rangle_{B_{i}}}{\sqrt{2}}, \\
\left|\Psi^{ \pm}\right\rangle_{A_{i} B_{i}} & =\frac{|0\rangle_{A_{i}}|1\rangle_{B_{i}} \pm|1\rangle_{A_{i}}|0\rangle_{B_{i}}}{\sqrt{2}},
\end{aligned}
$$




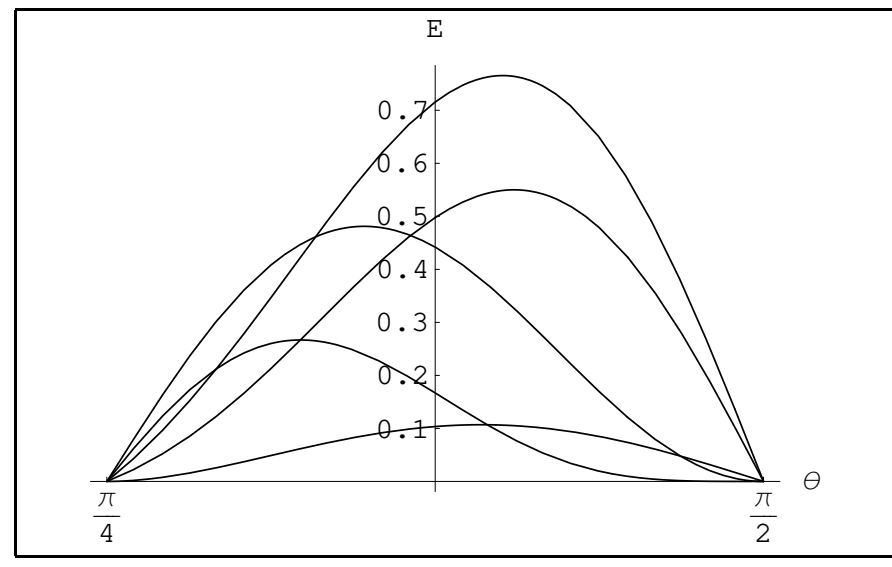

FIG. 1: Maximum efficiency $E(\theta)$ as a function of $\theta$ for different networks. Curves from bottom up refer to networks with number of nodes and number of entangled pairs equal to $(2,1)$, $(2,2),(3,3),(4,4),(5,5)$ respectively.

are the Bell states and $f$ is the fidelity between $w$ and $\left|\Phi^{+}\right\rangle$(notice that $0.25 \leq f \leq 1$ ). The initial state of the networks is

$$
W_{12 \ldots n}=w_{A_{1} B_{1}} \otimes w_{A_{2} B_{2}} \otimes \ldots \otimes w_{A_{n} B_{n}} .
$$

By using the same procedure shown for entanglement concentration, we can calculate the efficiency of the protocol. Networks with one or two qubits lead to a zero efficiency. In fact, the concurrence obtained after the evolution of the initial state in Eq. (16) is less than the initial one and it is identical for every outcomes. Actually for all outcomes $o$ we have

$$
C_{o}-C=\left(1+4 f-32 f^{2}\right) / 36 \leqslant 0 .
$$

The efficiency for three qubits becomes greater than zero and its maximum is represented in Fig. (2). Such maximum is obtained when $t=k \pi / \sqrt{2}, k \in \mathbb{N}$, in the open chain configuration (from (46) and when $t=k \pi$, $k \in \mathbb{N}$, in the fully connected configuration (from (48)).

Likewise the case of entanglement concentration, only the time $t$ at which maximum efficiency is attained is affected by the choice of the networks' topology (not the maximum efficiency itself), provided that there are no isolated nodes.

The maximum efficiency for $n=4$ and $n=5$ can be obtained from Eqs. (49) and (51), respectively. Their behavior is represented in Fig. (2).

\section{CONCLUDING REMARKS}

In conclusion, we have considered entanglement manipulation between two networks of spin-half particles with $X Y$-type interaction. We have related entanglement transmission to the fidelity achieved in state transfer when adopting the single excitation model.

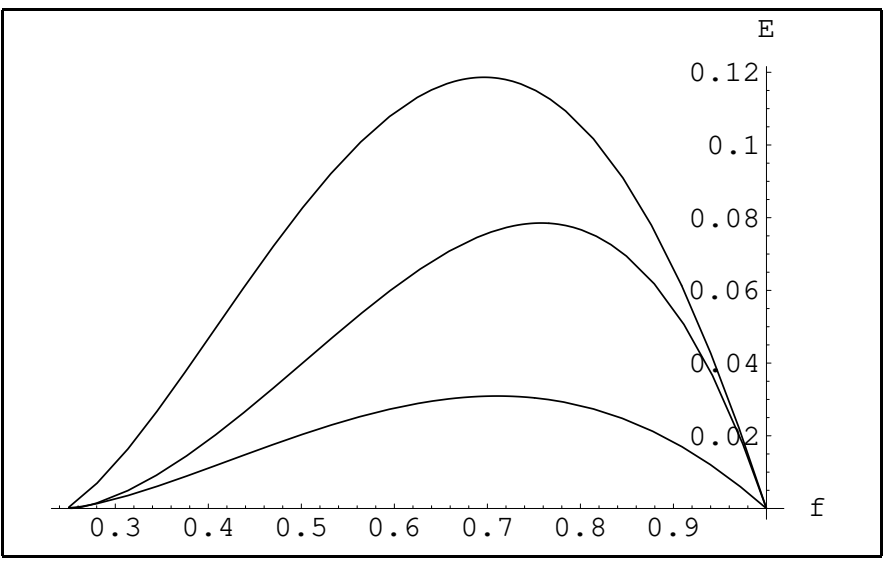

FIG. 2: Maximum efficiency $E(f)$ as a function of $f$ for different networks. Curves from bottom up refer to networks with number of nodes and number of entangled pairs equal to $(3,3),(4,4),(5,5)$ respectively.

We have shown how to increase the entanglement of pure and mixed states via free dynamics and local measurements on the nodes. The performance of the protocol are characterized by means of an efficiency measure. The performance appears to be independent of the networks' topology provided that there are no isolated nodes. The structure of the network only affects the time at which the maximum efficiency is attained. Moreover, we have determined the minimal number of initial entangled pairs shared between the two networks and needed to successfully complete the protocol (i.e., with nonzero efficiency). This number is 1 and 3 for entanglement concentration and purification, respectively. Furthermore, the results shown in Figs. (11) and (2) suggest that a (quasi) constant increase for the maxima of the efficiency is obtained with a corresponding increase for the number of entangled pairs. This can be estimated as about 0.15 , for the case of entanglement concentration, and about 0.04, for the case of purification for each additional pair. This trend suggests that a (quasi) deterministic protocol can be achieved, at least for specific values of $\theta$ or $f$, with a limited number of initially entangled pairs. Our protocol is straightforward and only based on the free dynamics occurring in the two networks. It may be an interesting venue to consider more general settings, as, for example, synchronous dynamics in different networks.

Notice that already in the single excitation model the XYZ (Heisenberg) interaction would give a different networks' dynamics. Actually a phase factor would be introduced in front of the state's components containing one excitation per network. However such a phase factor is not influent to our protocol as long as we consider measurements on the computational basis ( $\mathrm{Z}$ eigenvectors). Thus our conclusions can be applied to XY model as well as to Heisenberg model.

Finally, it is worth remarking that the proposed schemes for entanglement manipulation might be experimentally verified with networks containing few spins-half 
systems. Candidate physical systems would be semiconductor quantum dots [16], superconducting qubits [17] or atoms trapped in optical lattices [18].

\section{Acknowledgments}

While writing this paper, AC would like to thank Seth Lloyd for the kind of hospitality at Research Laboratory of Electronics (MIT) where part of this work was carried out. The work of SM is supported by the European Commission, under the FET-Open grant agreement HIP, number FP7-ICT-221889. SS is supported by a Newton International Fellowship. The authors would like to thank Yasser Omar for useful discussions.
[1] H. J. Kimble, Nature 453, 1023 (2008).

[2] W. Dür and H.-J. Briegel, Rep. Prog. Phys. 70, 1381 (2007); R. Horodecki, P. Horodecki, M. Horodecki, and K. Horodecki, quant-ph/0702225.

[3] M. Czechlewski, A. Grudka, S. Ishizaka and A. Wojcik, Phys. Rev. A 80, 014303 (2009); K. Fujii and K. Yamamoto, arXiv:0811.2639, M. Yang, F. Yan and Z. Cao, arXiv:0904.2343 S. Perseguers, arXiv:0910.1459. S. Perseguers, D. Cavalcanti, G. J. Lapeyre Jr, M. Lewenstein and A. Acin, arXiv:0910.2438.

[4] S. Perseguers, A. Acin, J. I. Cirac and M. Lewenstein, arXiv:0907.3283

[5] N. Paunkovic, Y. Omar, S. Bose and V. Vedral, Phys. Rev. Lett. 88, 187903 (2002).

[6] R. Blume-Kohout, S. Croke and D. Gottesman, arXiv:0910.5952

[7] K. Maruyama and F. Nori, Phys. Rev. A 78, 022312 (2008).

[8] D. Burgath, Eur. Phys. J. Special Topics, 151, 147 (2007); S. Bose, Contemp. Phys. 48, 13 (2007).

[9] M. Christandl, N. Datta, A. Ekert, A. Landahl, Phys. Rev. Lett. 92, 187902 (2004); M. Christandl, N. Datta, T. Dorlas, A. Ekert, A. Kay, A. Landahl, Phys. Rev. A
71, 032312 (2005); N. Saxena, S. Severini, and I. Shparlinski, Int. J. of Quant. Inf. 5, 417 (2007); S. Bose, A. Casaccino, S. Mancini, and S. Severini, Int. J. of Quant. Inf. 7, 713 (2009); A. Casaccino, S. Lloyd, S. Mancini and S. Severini, Int. J. of Quant. Inf. 7,1417 (2009).

[10] J. Lapeyre, J Wehr and M. Lewenstein, Phys. Rev. A 79, 042324 (2009).

[11] A. Acin, J. I. Cirac and M. Lewenstein, Nature Phys. 3, 256 (2007).

[12] M. Cuquet and J. Calsamiglia, arXiv:0906.2977.

[13] S. Broadfoot, U. Dorner and D. Jaksch, arXiv:0906.1622.

[14] R. F. Werner, Phys. Rev. A 40, 4277 (1989).

[15] W. Wooters, Phys. Rev. Lett. 80, 2245 (1998); W. Wooters and S. Hill, Phys. Rev. Lett. 78, 5002 (1997).

[16] D. Loss and D. P. DiVincenzo, Phys. Rev. A 57, 120 (1998); J. R. Petta, A. C. Johnson, J. M. Taylor, E. A. Laird, A. Yacoby, M. D. Lukin, C. M. Marcus, M. P. Hanson, and A. C. Gossard, Science 309, 2180 (2005).

[17] J. Q. You and F. Nori, Phys. Today 58, No. 11, 42 (2005).

[18] O. Mandel, M. Greiner, A.Widera, T. Rom, T.W. Hansch, and I. Bloch, Nature 425, 937 (2003). 